\title{
Il nuovo reato di falso in bilancio. Alle Sezioni Unite il compito di dirimere il contrasto interpretativo insorto nella Sezione V
}

\author{
Lorenzo Maria Corvucci
}

\section{Riassunto}

L'Autore torna in argomento sul nuovo reato di falso in bilancio delineato dall'art. 2621 c.c. per effetto dell'art. 9 L. n. $69 / 2015$.

Con Ordinanza Sez. 5 Num. 9186 Anno 2016 (Relatore Amatore Roberto), la Corte di Cassazione ha rimesso la questione alle Sezioni Unite ai sensi dell'art. 618 c.p.p. affinché le stesse si esprimano sul seguente quesito: "Se la modifica dell'art. 2621 c.c. per effetto dell'art. 9 L. n. 69/2015 nella parte in cui, disciplinando 'le false comunicazioni sociali', non ha riportato l'inciso 'ancorché oggetto di valutazioni', abbia determinato o meno un effetto parzialmente abrogativo della fattispecie".

Persiste infatti, sul punto, un contrasto interpretativo all'interno della medesima sezione $\mathrm{V}$ competente per materia. Nell'articolo si dà conto dei termini del contrasto.

\section{Résumé}

L'auteur examine une nouvelle fois le nouveau crime de faux en écriture comptable (article $n^{\circ} 621$ du Code Civil Italien) à cause de l'article $n^{\circ} 9$ de la loi $n^{\circ} 69 / 2015$.

Conformément à l'article $\mathrm{n}^{\circ} 618$ du Code de procédure pénale italien, sur ordonnance $\mathrm{n}^{\circ} 9168$ de 2016 , la cinquième Chambre de la Cour de Cassation Italienne a saisi les Sections Unies de la même Cour pour qu'elles puissent donner leur avis sur la question suivante : «si la modification de l'article $\mathrm{n}^{\circ} 2621$ a produit ou non un effet partiellement abrogatif du crime ».

En effet, un conflit d'interprétation sur ce point reste au sein de la cinquième Chambre.

L'article analyse en détail ce débat.

\section{Abstract}

The author reflects on the new offence of false accounting again (article $n^{\circ} 2621$ Italian Civil Code) because of article $n^{\circ} 9$ of Law $n^{\circ} 69 / 2015$.

According to article 618 of Italian Code of Criminal Procedure, by order n 9186 , year 2016, the Fifth Chamber of the Italian Court of Cassation has referred the matter to United Sections of the Court so that they can give their opinion "whether the modification of the article $n^{\circ} 2621$ has produced a recall effect on this crime or not".

In fact, a conflict of interpretation on this point remains even within the Fifth Chamber.

The article analyses this argument in detail.

Key words: false accounting; Italian Civil Code; crime; Italian Court of Cassation; conflict of interpretation.

\section{La questione devoluta ed i termini del} contrasto.

Con ordinanza resa il 9.2.2016 la sezione V Rel. Roberto Amatore - ha rimesso alle Sezioni Unite il seguente quesito: "Se la modifica dell'art. 2621 c.c. per effetto dell'art. 9 L. n. 69/2015 nella parte in cui, disciplinando 'le false comunicazioni sociali', non ha riportato l'inciso 'ancorché oggetto di valutazioni', abbia determinato o meno un effetto parzialmente abrogativo della fattispecie".

Le Sezioni Unite saranno chiamate a dare risposta il 30 marzo 2016.

Questi gli approdi interpretativi che si sono susseguiti all'indomani dell'entrata in vigore della novella L. n. 69/2015, che evidenziano il contrasto.

\footnotetext{
- Avvocato, Foro di Bologna.
} 
Il primo orientamento (cfr. sent. n. 33774 del 16/6/2015, dep. 30/7/2015, Crespi e altri) in tema di "bancarotta fraudolenta impropria 'da reato societario', di cui all'art. 223, secondo comma, n. 1, R.D. 16 marzo 1942 n. 267, ha evidenziato che la nuova formulazione degli artt. 2621 e 2622 cod. civ., introdotta dalla L. 27 maggio 2015, n. 69, ha determinato - eliminando l'inciso 'ancorché oggetto di valutazioni' ed inserendo il riferimento, quale oggetto anche della condotta omissiva, ai 'fatti materiali non rispondenti al vero' - una successione di leggi con effetto abrogativo, peraltro limitato alle condotte di errata valutazione di una realtà effettivamente sussistente"1.

Orbene, nel primo di tali interventi, la Corte ha rilevato che "il dato testuale e il confronto con la previgente formulazione degli artt. 2621 e 2622, come si è visto in una disarmonia con il diritto penale tributario e con l'art. 2638 cod. civ., sono elementi indicativi della reale volontà legislativa di far venir meno la punibilità dei falsi valutativi".

Si evidenzia, nell'ordinanza di rimessione, che "A fondamento di tale affermazione, si è ritenuto che il legislatore del 2015 ha ripreso la formula utilizzata dal legislatore del 2002 'fatti materiali', diversa da quella 'fatti' contenuta nell'originario art. 2621 cod. civ., per circoscrivere l'oggetto della condotta attiva, privandola tuttavia del riferimento alle valutazioni e provvedendo contestualmente a replicarla anche nella definizione di quello della condotta omissiva, in relazione alla quale il testo

\footnotetext{
${ }^{1}$ Tale orientamento è stato oggetto di disamina in questa Rivista Vol. IX - N. 3 - SettembreDicembre 2015 - Articolo: "Il nuovo reato di false comunicazioni sociali" - di Lorenzo Maria Corvucci.
}

previgente faceva invece riferimento, come detto, alle 'informazioni"'.

Argomenta infatti la sentenza Crespi che il disegno di legge n. 19 prevedeva di attribuire, in un primo momento, rilevanza alle "informazioni" false, adottando così un'espressione lessicale idonea a ricomprendere le valutazioni, sicché proprio tale mutamento sarebbe espressivo della intenzione del legislatore di escludere la rilevanza penale del c.d. falso valutativo.

In un contesto in cui "l'espressione 'fatti materiali’ era stata già utilizzata dalla legge n. 154 del 1991 per circoscrivere l'oggetto del reato di frode fiscale di cui all'art. 4 lett. f) della legge $n$. 516 del 1982, con il chiaro intento di escludere dall'incriminazione le valutazioni relative alle componenti attive e passive del reddito dichiarato. Invero, il citato art. 4, lett. f), puniva l'utilizzazione di 'documenti attestanti fatti materiali non corrispondenti al vero', nonché il compimento di 'comportamenti fraudolenti idonei ad ostacolare l'accertamento di fatti materiali"”.

Si osserva pertanto che "pacificamente una tale formulazione del dato normativo comportava l'irrilevanza penale di qualsiasi valutazione recepita nella dichiarazione dei redditi, in quanto ciò fu conseguenza di una scelta legislativa ben esplicitata nel disegno di legge e con la quale si vollero evitare conseguenze penali da valutazioni inadeguate o comunque in qualche modo discutibili alla luce della complessa normativa tributaria".

Altra annotazione riguarda la circostanza che i testi riformati degli artt. 2621 e 2622 cod. civ. si inseriscono in un contesto normativo che vede ancora un esplicito riferimento alle valutazioni 
nell'art. 2638 cod. civ. ("Ostacolo all'esercizio delle funzioni delle autorità pubbliche di vigilanza"). Tale disposizione continua invero a punire i medesimi soggetti attivi ("gli amministratori, i direttori generali, i dirigenti preposti alla redazione dei documenti contabili societari, i sindaci e i liquidatori di società....”) dei reati di cui agli artt. 2621 e 2622 che, nelle comunicazioni dirette alle autorità pubbliche di vigilanza, "espongono fatti materiali non rispondenti al vero, ancorché oggetto di valutazioni".

Secondo tale esegesi "una lettura ancorata al canone interpretativo 'ubi lex voluit dixit, ubi noluit tacuit non può trascurare la circostanza dell'inserimento di modifiche normative in un sistema che riguarda la rilevanza penale delle attività societarie con una non giustificata differenziazione dell'estensione della condotta tipizzata in paralleli ambiti operativi, quali sono quelli degli articoli 2621 e 2622 cod. civ., da una parte, e art. 2638 cod. civ, dall'altra, norme che, sebbene tutelino beni giuridici diversi, sono tutte finalizzate a sanzionare la frode nell'adempimento dei doveri informativi".

Il secondo orientamento, contrapposto al primo, è rappresentato dalla sentenza n. 890/2016 Ric. Giovagnoli2.

2 Di tale sentenza, ai tempi della redazione dell'Articolo di cui a Nota 1, era disponibile solo l'informazione provvisoria N. 13/2015 - Udienza P.U. 12.11.2015 che, sulla questione in esame, aveva adottato soluzione affermativa, per cui "Nell'art. 2621 c.c. il riferimento ai 'fatti materiali' quali possibili oggetti di una falsa rappresentazione della realtà non vale a escludere la rilevanza penale degli enunciati valutativi, che sono anch'essi predicabili di falsità quando violino criteri di valutazione predeterminati o esibiti in una comunicazione sociale. Infatti, quando intervengano in contesti che implicano l'accettazione di parametri di valutazione
Secondo la Corte, la rimozione dal testo previgente della locuzione "ancorché oggetto di valutazione" non viene ad assumere alcuna valenza decisiva.

Rileva infatti il Collegio, in questo caso, che la congiunzione "ancorché" riveste una finalità ancillare, meramente esplicativa e chiarificatrice del nucleo sostanziale della proposizione principale, con la conseguenza che il suo significato si coglie in funzione della precisazione - ritenuta opportuna per fugare dubbi interpretativi - che nei "fatti materiali" - oggetto di esposizione nei bilanci, nelle relazioni e nelle altre comunicazioni sociali - siano da intendersi ricompresi anche quelli che sottendono attività valutative.

$\mathrm{Ne}$ consegue che, secondo questa tesi, la predetta proposizione concessiva avrebbe solo una funzione prettamente esegetica e sicuramente non additiva.

L'ordinanza di rimessione si sofferma su tale orientamento, così sintetizzato: “ $[\ldots]$ la Corte passa ad esaminare il significato della locuzione 'fatti materiali rilevanti', osservando che i termini 'materiali rilevanti' sono di natura squisitamente tecnica, frutto di una trasposizione letterale di formule lessicali in uso nelle scienze economiche anglo-americane e nella legislazione comunitaria. $\mathrm{Ne}$ discende che la qualificazione 'materiale' si connetterebbe, sempre secondo questa opzione esegetica, al concetto tecnico di 'materialità' (o 'materiality') che da tempo è stato adottato dagli economisti anglo-americani come criterio di redazione dei bilanci di esercizio e di revisione.

normativamente determinati o, comunque, tencicamente indiscussi, lgi enunciati valutativi sono idonei ad assolvere una funzione informativa e possono dirsi veri o falsi". 
Si evidenzia pertanto che il termine in esame va qualificato come sinonimo di 'essenzialità' e ciò nel senso che nel bilancio devono entrare solo dati informativi 'essenziali' ai fini dell'informazione, restandone fuori tutti i profili marginali e secondari, in aderenza a quanto stabilito dalla legislazione comunitaria (art. 2, comma terzo, della IV direttiva CEE sul bilancio di esercizio e art. 16, comma terzo, della VII Direttiva CEE sul bilancio consolidato ) e dalla legislazione nazionale all'art. 2423 c.c.”.

Allo stesso modo ritiene la Corte, nella sentenza qui richiamata, che l'aggettivo "rilevante" è di stretta derivazione dal lessico della normativa comunitaria, riconnettendosi al concetto di rilevanza sancito dall'art. 2, punto 16, della direttiva 2013/34/UE, e realizzandosi "quando la omissione o errata indicazione del fatto rilevante potrebbe ragionevolmente influenzare le decisioni prese dagli utilizzatori sulla base del bilancio di impresa". Con la precisazione tuttavia che la 2rilevanza delle singole voci è giudicata nel contesto di altre voci analoghe" e con la ulteriore conseguenza che deve ritenersi pertanto normativamente introdotto nel nostro ordinamento un nuovo principio di redazione del bilancio e cioè proprio quello della rilevanza. $\mathrm{Ne}$ consegue ancora che "materialità" e "rilevanza" dei fatti economici da rappresentare in bilancio costituirebbero facce della stessa medaglia, postulando un'indicazione di indefettibile "corretta" informazione e che le aggettivazioni "materiali" e "rilevanti", lungi dal costituire una ridondante endiadi, dovrebbero trovare senso compiuto nella loro genesi, connessa come tale alla funzione di corretti veicoli di informazioni capaci di orientare le scelte operative e le decisioni strategiche dei destinatari . Pertanto, in tale contesto ermeneutico - secondo l'arresto da ultimo ricordato - anche il lemma "fatto" non potrebbe essere inteso nel significato comune, ossia come fatto/evento fenomenologico, quanto piuttosto nella sua accezione tecnica più lata di dato informativo della realtà che i bilanci e le altre comunicazioni sono destinati a proiettare all'esterno, con la conseguente irrilevanza, sul piano della interpretazione sistematica della norma, della soppressione - intervenuta nel corso dei lavori preparatori - del termine "informazioni" e del rispristino dell'originario lemma "fatti". Peraltro, la Corte - in questo pronunciamento - ritiene quanto mai significativo il riferimento alle norme di cui ai nuovi artt. 2621-bis c.c. ("Fatti di lieve entità"), che prevede una diminuzione di pena ove i fatti di cui all'art. 2621 c.c. siano di lieve entità, "tenuto conto della natura e delle dimensioni della società e delle modalità o degli effetti della condotta" e nel caso in cui i fatti di cui all'art. 2621 riguardino società che non superino i limiti indicati dal secondo comma dell'art. 1 R.D. 16 marzo 1942, n. 267, ai fini della fallibilità; e 2621ter c.c. ("Non punibilità per particolare tenuità"), che stabilisce che, ai fini dell'applicabilità, in materia, della causa di non punibilità per particolare tenuità di cui all'art. 131 bis cod. pen., il giudice deve considerare "in modo prevalente, ... l'entità dell'eventuale danno cagionato alla società, ai soci o ai creditori conseguente ai fatti di cui agli artt. 2621 e 2621 bis". Argomenta così la Corte che l'utilizzo del criterio della rilevanza fa da contrappeso all'eliminazione delle soglie di punibilità e del riferimento esplicito alle valutazioni estimative che figurava nella precedente formulazione degli artt. 2621 e 2622 
cod. civ. e riafferma il potere discrezionale del giudice in materia di accertamento del coefficiente di significatività della falsa rappresentazione, da apprezzarsi in concreto al di là di ogni predeterminazione positiva in termini quantitativi.

Conclude pertanto la Corte che nella nozione di rappresentazione di fatti materiali e rilevanti non possano non ricomprendersi anche le valutazioni, giacché le valutazioni espresse in bilancio non sono il frutto di mere congetture od arbitrari giudizi di valore, ma devono uniformarsi a criteri valutativi positivamente determinati dalla disciplina civilistica (art. 2426 c.c.), dalle direttive e regolamenti di diritto comunitario o da prassi contabili generalmente accettate. Con la conseguenza che il mancato rispetto di tali parametri comporta la falsità della rappresentazione valutativa, ancor'oggi punibile ai sensi del nuovo art. 2621 cod. civ., nonostante la soppressione dell'inutile inciso "ancorché oggetto di valutazione".

Peraltro, osserva ulteriormente la Corte, per la ricerca di momenti di conferma del principio affermato, in chiave di interpretazione teleologica, che anche l'inserimento sistematico delle nuove false comunicazioni sociali in un testo normativo "anticorruzione" deporrebbe nel senso di rendere irragionevole l'esclusione dall'alveo dei falsi punibili anche quelli valutativi perché, diversamente opinando, verrebbe frustrata la finalità complessiva della legge. Chiarisce inoltre che - in ragione della circostanza che la stragrande maggioranza delle voci in bilancio è frutto di una qualche valutazione - una diversa esegesi del dato normativo si risolverebbe in una interpretatio abrogans della fattispecie penale in esame.
Nella sentenza Giovagnoli si contrasta esplicitamente anche l'argomento tratto, dalle altre sentenze, dal testo dell'art. 2638 c.c. nel quale è mantenuto il sintagma "ancorché oggetto di valutazioni", con riferimento ai fatti materiali non rispondenti al vero, oggetto delle comunicazioni di legge alle autorità pubbliche di vigilanza, testo da quelle valutato alla stregua del canone interpretativo "ubi lex voluit dixit, ubi noluit tacuit". Ha rilevato cioè la Corte che il ricorso a criteri logici di comparazione può aspirare ad un obiettivo di ragionevole affidabilità solo in presenza di identità delle fattispecie di riferimento, ove invece quelle in esame (rispettivamente previste dagli artt. 2621 e 2638 cod. civ.) hanno natura ed obiettività giuridiche diverse e perseguono finalità radicalmente differenti.

D'altronde, prosegue, se non si dovesse tener ferma la diversità dei beni giuridici tutelati dalla richiamate fattispecie delittuose e fosse, viceversa, praticabile la tesi opposta, si avrebbe il risultato paradossale - e forse di dubbia costituzionalità - che la redazione di uno stesso bilancio, recante falsi valutativi, sarebbe penalmente irrilevante se diretto ai soci ed al pubblico e penalmente rilevante se rivolto alle autorità pubbliche di vigilanza.

$\mathrm{Si}$ è anche ricordato che questa Corte di legittimità, già in passato, ha avuto modo di statuire che il reato di ostacolo all'esercizio delle funzioni delle autorità pubbliche di vigilanza è configurabile anche nel caso in cui la falsità sia contenuta in giudizi estimativi delle poste di bilancio, "atteso che dal novero dei 'fatti materiali', indicati dall'attuale norma incriminatrice come possibile oggetto della falsità, vanno escluse soltanto le previsioni o 
congetture prospettate come tali, vale a dire quali apprezzamenti di carattere squisitamente soggettivo, e l'espressione, riferita agli stessi fatti, 'ancorché oggetto di valutazioni', va intesa in senso concessivo, per cui, in ultima analisi, l'oggetto della vigente norma incriminatrice viene a corrispondere a quello della precedente, che prevedeva come reato la comunicazione all'autorità di vigilanza di 'fatti non corrispondenti al vero"” (Sez. 5, n. 44702 del 28 settembre 2005, Rv 232535; nello stesso senso, recentemente, Sez. 5, n. 49362 del 7 dicembre 2012, Rv 254065).

Nell'occasione, questa Corte ha, dunque, statuito che l'espressione "fatti materiali, ancorché oggetto di valutazioni” coincideva con quella di "fatti non corrispondenti al vero", cioè sostanzialmente con il testo dell'originario art. 2621 cod. civ., così offrendo significativo riscontro all'interpretazione secondo cui il sintagma introdotto con la I. n. 61 del 2002 era mera superfetazione.

Nella medesima sentenza si sottolinea poi che, con riferimento al testo dell'art. 2621 c.c. antecedente alla riforma del 2002 - quello cioè plasmato dal legislatore del 1942 il quale non aveva fatto alcun riferimento esplicito alla nozione di "valutazioni" - si era formata una giurisprudenza che nessun dubbio aveva rammostrato nel riconoscere la idoneità del precetto a coprire anche condotte riconducibili ad attività valutative: così Sez. 5, n. 234 del 16/12/1994, Rv. 200455, secondo cui in tema di false comunicazioni sociali, art. 2621 cod. civ., la veridicità o falsità delle componenti del bilancio va valutata in relazione alla loro corrispondenza ai criteri di legge e non alle enunciazioni "realistiche" con le quali vengono indicate.
Si cita anche, nella medesima prospettiva, l'orientamento consolidato in tema di reati di falso secondo cui anche la valutazione, quando non corrisponda al vero, può essere falsa (Sez. 5, n. 1004 del 30/11/1999, dep. 2000, Rv. 215744). In altri termini, nell'ambito di determinati contesti che implichino l'accettazione di parametri valutativi normativamente determinati o tecnicamente indiscussi, le valutazioni formulate da soggetti cui la legge riconosce una determinata perizia possono non solo configurarsi come errate, ma possono rientrare altresì nella categoria della falsità: ciò in quanto, laddove il giudizio faccia riferimento a criteri predeterminati, esso è un modo di rappresentare la realtà analogo alla descrizione o alla constatazione (enunciati pacificamente falsificabili, quantunque, rispetto a tali categorie della conoscenza logica, esso dipenda in maggior misura dal grado di specificità dei criteri di relazione). Ne consegue, pertanto, che può dirsi falso l'enunciato valutativo che contraddica criteri indiscussi o indiscutibili e sia fondato su premesse contenenti false attestazioni (Sez. 5, n. 3552 del 9/2/1999, Rv. 213366)].

A tale orientamento ne è seguito altro che si è posto sulla stessa linea della sentenza Crespi (Penale Sent. Sez. 5 Num. 6916 Anno 2016 Presidente: Zaza Carlo - Relatore: Amatore Roberto - Data Udienza: 8/1/2016 - Ric. Banca Popolare dell'Alto Adige soc. coop. $)^{3}$, con argomentazioni pressoché coincidenti.

\footnotetext{
${ }^{3}$ Così in premessa Sent. Sez. 5 Num. 6916 Anno 2016: "ritiene questo Collegio giudicante che occorra fornire continuità applicativa al principio già fissato da questa Corte con il noto precedente rappresentato dalla sentenza $n .33774$ del 16/6/2015 (dep. 30/7/2015, Crespi e altri, Rv. 264868). Ed invero, ̀̀ stato convincentemente affermato, nel ricordato arresto giurisprudenziale,
} 
Da qui la rimessione alle Sezioni Unite.

La riforma, nei termini evidenziati, ha determinato o meno un effetto parzialmente abrogativo della fattispecie?

\section{Dibattito in corso, in attesa della}

\section{decisione delle Sezioni Unite.}

La questione specifica qui in esame, stante l'importanza, ha generato un proliferare di interventi, a tutti i livelli: ambito dottrinaleaccademico; ambito professionale; ambito stampa specializzata.

Si susseguono Convegni in argomento, articoli, commenti, note a sentenza.

Il contrasto giurisprudenziale, e comunque le difficoltà che avrebbe incontrato la Corte di Cassazione nella lettura interpretativa del nuovo testo consegnato dalla novella, che vede l'espunzione della formula "ancorché oggetto di valutazioni" - in sostanza se, con le modifiche normative, permanga la rilevanza penale del c.d. falso valutativo - era ampiamente prevedibile, se non già messo in conto.

il principio interpretativo secondo il quale in tema di bancarotta fraudolenta impropria "da reato societario", di cui all'art. 223, secondo comma, $n$. 1, R.D. 16 marzo 1942 n. 267, la nuova formulazione degli artt. 2621 e $2622 \mathrm{cod}$. civ., introdotta dalla L. 27 maggio 2015, n. 69, ha determinato - eliminando l'inciso "ancorché oggetto di valutazioni", ed inserendo il riferimento, quale oggetto anche della condotta omissiva, ai "fatti materiali non rispondenti al vero" - una successione di leggi con effetto abrogativo, peraltro limitato alle condotte di errata valutazione di una realtà effettivamente sussistente (in applicazione del principio, la S.C. ha ritenuto escluse dall'effetto parzialmente abrogativo l'esposizione di crediti inesistenti perché originati da contratti fittizi, l'esposizione di crediti concernenti $i$ ricavi di competenza dell'esercizio successivo, l'esposizione di crediti relativi ad una fattura emessa per operazioni inesistenti )...”.
Lo abbiamo già evidenziato nel citato articolo ${ }^{4}$, ove, nel dibattito parlamentare, il Relatore riferiva espressamente che di tale questione se ne sarebbe occupata la Corte di Cassazione e non certo il Parlamento (sic il Relatore di maggioranza al Senato).

Tale posizione rende oltre modo arduo per la Corte, organo di nomofilachia, comprendere le reali intenzioni del legislatore, sol se si considera che lo stesso se ne disinteressa espressamente, denegando ogni intervento chiarificatore ed esplicativo di indirizzo.

Quale contributo può dunque venire in soccorso all'interprete dalla lettura dei lavori parlamentari? Nessuno.

Il legislatore è tenuto ad una stretta osservanza dei principi di tassatività e determinatezza della norma incriminatrice interna, pena redazione di un testo legislativo oscuro ed imperfetto.

In un contesto in cui, sebbene $\mathrm{i}$ riferimenti evincibili dalla lettura dei lavori parlamentari non possano, ex se, in alcun modo, ritenersi decisivi al fine di individuare una corretta interpretazione logico-sistematica del contenuto della autonoma norma incriminatrice, tuttavia è indubitabile che gli stessi rappresentino un utile ingrediente della operazione di ricostruzione della voluntas legis.

${ }^{4}$ In questa Rivista Vol. IX - N. 3 - SettembreDicembre 2015, Corvucci L. M., "Il nuovo reato di false comunicazioni sociali": Così, infatti, testualmente afferma il Relatore nel corso della seduta n. 420 del 31/3/2015 (Assemblea Senato): "La nostra Corte di Cassazione dovrà valutare se gli elementi valutativi e le stime possano o meno rientrare allinterno di un concetto che implica fatti materiali rilevanti. Ripeto, il relatore a tal proposito non dà alcuna risposta, perché non la deve dare; ci mancherebbe altro che la desse. Noi non siamo la Corte di Cassazione che dà risposte giurisprudenziali; questo è un tema che la giurisprudenza affronterà".

5 Bricchetti R., "Alla ricerca di una 'terza via' chiara e determinata", Guida al Diritto, n. 14, 26 
E' un fatto che la giurisprudenza di legittimità, in sede di operazione ermeneutica volta all'interpretazione della legge, nei casi in cui si renda necessaria, attinga proprio ai lavori parlamentari. Ciò non sarà utile nel caso di specie. La chiave di lettura va dunque ricercata aliunde.

Nel compiere tale operazione - in ossequio al generale principio di cui all'art. 12 delle cd. preleggi (peraltro rafforzato nella sua valenza dalla materia penalistica) - va tenuta presente la collocazione "topografica" della norma incriminatrice e va altresì operata una lettura non limitata al singolo termine utilizzato ma estesa all'intera proposizione.

Crediamo che le Sezioni Unite, al pari sia della sentenza Crespi, favorevole ad un'abrogazione parziale della norma incriminatrice, sia della sentenza Giovagnoli, contraria, con interpretazione estensiva, nella lettura del significato letterale della norma, muoveranno dall'analisi dell'art. 12 preleggi e non potrebbe essere diversamente.

La connessione delle parole (per stare nel solco tracciato dal citato articolo 12) è infatti spesso rivelatrice del reale significato del termine prescelto per descrivere una condotta, lì dove come nel caso in esame - il dato semantico può dì per sé essere non chiaro. Ma un dubbio permane.

marzo 2016: "Soppressione voluta (n.d.a.: si riferisce all'inciso "ancorché oggetto di valutazioni") (l'intenzione del legislatore, dunque, c'è); perché voluta non lo si sa. Si sa, però, che il relatore di maggioranza al senato, espressamente richiesto di esprimersi in ordine agli effetti di detta amputazione, ha dichiarato che sarà "la nostra Corte di cassazione a dover valutare se gli elementi valutativi e le stime possano o meno rientrare all'interno di un concetto che implica fatti materiali rilevanti".
La norma sembra infatti, nel caso di specie, compiutamente descritta, lì dove il legislatore non ha scientemente riportato l'inciso "ancorchè oggetto di valutazioni”".

Dunque, una prima lettura legittima quanto evidenziato nella sentenza Crespi: "il dato testuale e il confronto con la previgente formulazione degli artt. 2621 e 2622, come si è visto in una disarmonia con il diritto penale tributario e con l'art. 2638 cod. civ., sono elementi indicativi della reale volontà legislativa di far venir meno la punibilità dei falsi valutativi".

Tale orientamento assegna valore assoluto all'argomento letterale secondo il brocardo $u b i$ lex voluit dixit ubi noluit tacuit, accordando valore dimostrativo alla soppressione dell'inciso “ancorché oggetto di valutazioni”.

Tanto anche in considerazione della eliminazione della soglia delle valutazioni estimative ed il mantenimento, al contempo, della medesima formula nel testo dell'art. 2638 del codice civile, norma che configura il delitto di ostacolo alle autorità pubbliche di vigilanza.

$\mathrm{Si}$ registrano annotazioni critiche su tale argomento ${ }^{6}$.

\footnotetext{
6 "Non mi convince della prima ipotesi il valore assoluto attribuito all'argomento letterale dell'ubi voluit dixit evocato per dimostrare il valore della soppressione, unita alla non riproposizione della soglia $(10 \%)$ delle valutazioni estimative, a fronte del mantenimento della formula nel testo dell'art. 2638 del codice civile (tale disposizione - nella quale è configurato il delitto di ostacolo alle autorità pubbliche di vigilanza - continua infatti a punire i medesimi autori dei reati in commento che, nelle comunicazioni dirette alle suddette autorità, "espongono fatti materiali non rispondenti al vero, ancorchè oggetto di valutazioni"), norma che negli anni del post riforma 2002 ha occupato alcuni degli spazi lasciati scoperti dall'annientamento della fattispecie di false comunicazioni sociali. L'argomento letterale non è, nell'attività
} 
Per converso tale conclusione è duramente criticata nella sentenza Giovagnoli la quale, infatti, porterebbe al risultato paradossale (n.d.a. certamente non perseguito dal legislatore) in cui uno stesso bilancio, recante falsi valutativi, sarebbe penalmente irrilevante se diretto ai soci ed al pubblico e penalmente rilevante se rivolto alle autorità pubbliche di vigilanza.

Sennonché la sentenza Giovagnoli muove certamente da un corretto presupposto: "L'esegesi della norma dovrà, ovviamente, essere condotta secondo gli ordinari canoni ermeneutici dettati dall'art. 12 preleggi, secondo cui 'nell'applicare la legge non si può ad essa attribuire altro senso che quello fatto palese dal significato proprio delle parole secondo la connessione di esse e dallintenzione del legislatore', quest'ultima da intendersi - per quanto si è detto - in termini rigorosamente oggettivi, come volontà 'consacrata nel dettato normativo" (così si legge a pag. 8), ..."Solo in via sussidiaria, in caso di ambiguità del dato testuale, è consentito il ricorso ad altri parametri interpretativi di supporto..”, con l'avvertenza e precisazione che "..all'ineludibile indagine testuale debba associarsi il richiamo al canone logico-sistematico ed a quello teleologico, ai fini della compiuta focalizzazione dell'impatto della novella sull'assetto normativo preesistente" (così

interpretativa, necessariamente prevalente su quello logico. Un'interpretazione letterale inadeguata non può, ad esempio, che lasciare spazio all'interpretazione che valorizza l'inequivoca interpretazione del legislatore. D'altra parte l'articolo 12 delle cosidette "preleggi", che detta regole (non tutte) dell'interpretazione, non dice, per il caso in conflitto, quale dei due parametri (verba o mens) debba prevalere. Lascia all'interprete la soluzione dell'eventuale problema"; così ancora Bricchetti R., "Alla ricerca di una 'terza via' chiara e determinata", Guida al Diritto, n. 14, 26 marzo 2016. a pag. 9), salvo poi ritenere la soppressione della locuzione "ancorché oggetto di valutazioni" quale inutile inciso, che non viene ad assumere alcuna decisiva rilevanza in sede interpretativa?. Sul piano letterale, dunque, secondo la sentenza Giovagnoli, tale locuzione rileverebbe quale “tipica proposizione 'concessiva' introdotta da congiunzione (ancorché) notoriamente equipollente ad altre tipiche e similari ('sebbene', 'benché', 'quantunque', 'anche se' et similia). Ed è risaputo che una proposizione siffatta ha finalità ancillare, meramente esplicativa e chiarificatrice del nucleo sostanziale della proposizione principale. Nel caso di specie, il suo precipuo significato si coglie in funzione della precisazione - ritenuta opportuna, onde fugare possibili dubbi (agitati in sede interpretativa) - che nei 'fatti materiali' oggetto di esposizione nei bilanci, nelle relazioni o nelle altre comunicazioni sociali dirette ai soci o al pubblico, sono da intendersi ricompresi anche quelli oggetto di valutazione.

La proposizione concessiva ha, dunque, funzione prettamente esegetica e, di certo, non additiva, di talché la sua soppressione nulla può aggiungere o togliere al contesto semantico di riferimento.

\footnotetext{
${ }^{7}$ Ancora Bricchetti R., "Alla ricerca di una "terza via' chiara e determinata”, Guida al Diritto, n. 14, 26 marzo 2016: "Neppure mi convince l'altra ipotesi che archivia la soppressione come eliminazione di un inciso inutile. Dimentica "la storia" della disposizione, archivia come sprovveduto il legislatore dell'aggettivo "materiali" e dell'inciso "incriminato" (che tratta come se non ci fossero, o meglio come se fosse scritta solo la parola "fatti"; difficile pensarla diversamente: l'accostamento di "materiali" all'inciso ne determina la reciproca elisione), ma ci consegna un legislatore del 2015 niente affatto sprovveduto, anzi molto colto, al punto di cogliere quanto fosse stato insulso il suo predecessore del 2002 che quell'inciso aveva inserito".
} 
Conseguentemente, nel caso di specie, l'elisione di una proposizione siffatta non può, certo, autorizzare la conclusione che si sia voluto immutare l'ambito sostanziale della punibilità del falsi materiali, che, invece, resta impregiudicata, continuando a ricomprendere, come in precedenza, anche i fatti oggetto di mera valutazione. In sostanza, l'intervento in punta di penna del legislatore ha inteso 'alleggerire' il precipitato normativo, espungendo una precisazione reputata superflua, siccome mera superfetazione linguistica...".

Sul piano logico-sistematico, invece, i medesimi giudici di legittimità (sentenza Giovagnoli) colgono “..l'ininfluenza della rimodulazione normativa" esprimendo un principio di diritto, a compendio delle motivazioni esposte, “.. secondo cui nell'art. 2621 cod. civ. il riferimento ai 'fatti materiali' oggetto di falsa rappresentazione non vale a escludere la rilevanza penale degli enunciati valutativi, che sono anch'essi predicabili di falsità quando violino criteri di valutazione predeterminati. Infatti, qualora intervengano in contesti che implichino accettazione di parametri di valutazione normativamente determinati o, comunque, tecnicamente indiscussi, anche gli enunciati valutativi sono idonei ad assolvere ad una funzione informativa e possono, quindi, dirsi veri o falsi”.

Si tratta però di interpretazione della norma di scopo, creativa, improntata certamente a

\footnotetext{
8 Ancora Bricchetti: "E pensare che la sentenza Giovagnoli ricorda l'articolo 12 delle preleggi ("L'esegesi della norma dovrà, ovviamente, essere condotta secondo gli ordinari canoni ermeneutici dettati dall'articolo 12 preleggi"); dopo avere predicato bene però, forse a causa di quell"'ovviamente" (che di solito si usa quando di “ovvio" non c'è nulla), "razzola" male".
}

ragionevolezza per non depotenziare la norma, che non si crede possa però, sol per questo, essere avallata, sic et simpliciter, dalle Sezioni Unite. E' un fatto che le nuove fattispecie non richiamino in alcun modo le valutazioni come oggetto di falsità; ciò dovrebbe integrare - in base alla legge vigente - ipotesi di abolitio criminis (art. 2 codice penale), in considerazione di una discontinuità normativa rispetto alla disciplina previgente 9.

Si ritiene infatti che il disvalore espresso dalla condotta penalmente sanzionata debba essere individuato esclusivamente all'interno della cornice normativa che la descrive che deve essere a sua volta applicata in conformità ai principi di stretta legalità, tassatività e determinatezza che governano l'interpretazione della legge penale, onde evitare di pervenire a non ammesse interpretazioni analogiche volte a ricomprendere nella fattispecie penale elementi normativi ("valutazioni") che la norma stessa non configura ai fini della punibilità dell'autore. Gli elementi normativi della fattispecie sono infatti parte integrante di una norma.

In definitiva, a mio avviso, i giudici della sentenza Giovagnoli compiono

\footnotetext{
9 Lanzi A., "Quello strano scoop del falso in bilancio che torna reato", Guida al Diritto, n. 26, 20 giugno 2015: "Le nuove fattispecie, infatti, non richiamano in alcun modo le valutazioni come oggetto di falsità; sul punto (salvo casi di interpretazione tanto creativa quanto contra legem) si è dunque realizzata una chiara ipotesi di abolitio criminis per discontinuità normativa (quanto prima era espressamente previsto come illecito dalla norma penale più non è menzionato dalla nuova norma penale. Il tutto con le conseguenze di cui all'art. 2, comma 2, del Cp anche in relazione alle condanne nel frattempo intervenute per bilanci contenenti false valutazioni di rilevanza penale quantitativa, predeterminata dalla legge in allora vigente, che non fossero però sussumibili in fatti materiali di per sé già penalmente rilevanti”.
} 
un'interpretazione correttiva attraverso

un'analogia in malam partem vietata.

Se una norma è oscura dovrà intervenire nuovamente il legislatore oppure la Corte Costituzionale, se investita. Non si crede, però, che le Sezioni Unite avranno l'umiltà di investire la Consulta.

Certamente una norma di difficile interpretazione (ripetesi oscura) non diventerà chiara e di lineare lettura solo attraverso un'operazione interpretativa da parte della giurisprudenza di legittimità.

E nel frattempo? Può permettersi il nostro Paese un periodo di vacatio in un settore tanto delicato? Occorrerebbe rammentare che sono pendenti, in fase dibattimentale, importanti processi che vedono sul banco degli imputati soggetti apicali nel settore bancario quali responsabili, in ipotesi d'accusa, dei reati di cui trattasi.

Alle Sezioni Unite, dunque, l'ardua sentenza ${ }^{10}$.

\section{Bibliografia.}

10 Nelle more tra la trasmissione del presente articolo e la pubblicazione si sono pronunciate sul tema le Sezioni Unite. E' disponibile solo l'informazione provvisoria, della quale occorre dare conto. L'autore si riserva gli approfondimenti del caso non appena saranno disponibili le motivazioni. Sulla nota questione controversa, "Se, ai fini della configurabilità del delitto di false comunicazioni sociali, abbia tuttora rilevanza il falso 'valutativo' pur dopo la riforma di cui alla legge n. 69 del 2015", le Sezioni Unite hanno dato risposta affermativa stabilendo che "Sussiste il delitto di false comunicazioni sociali, con riguardo alla esposizione o alla omissione di fatti oggetto di 'valutazione', se, in presenza di criteri di valutazione normativamente fissati o di criteri tecnici generalmente accettati, l'agente da tali criteri si discosti consapevolmente e senza darne adeguata informazione giustificativa, in modo concretamente idoneo ad indurre in errore $\mathrm{i}$ destinatari delle comunicazioni" [Nota a cura del coordinatore del Comitato editoriale].
- Bricchetti R., "Alla ricerca di una 'terza via' chiara e determinata”, Guida al Diritto, n. 14, 26 marzo 2016.

- Corvucci L.M., "Il nuovo reato di false comunicazioni sociali", Rivista di Criminologia, Vittimologia e Sicurezza, vol. IX, n. 3, settembre-dicembre 2015.

- Lanzi A., "Quello strano scoop del falso in bilancio che torna reato", Guida al Diritto, n. 26, 20 giugno 2015. 